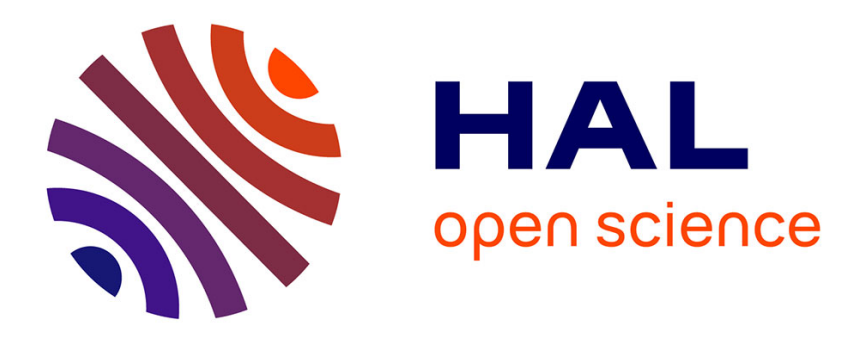

\title{
Estimation du coefficient de régression enfant/parent de quelques caractères du pêcher dans le cas de familles issues d'autofécondations
}

\author{
René Monet, Yves Bastard
}

\section{- To cite this version:}

René Monet, Yves Bastard. Estimation du coefficient de régression enfant/parent de quelques caractères du pêcher dans le cas de familles issues d'autofécondations. Agronomie, 1982, 2 (4), pp.347-358. hal-00884391

\section{HAL Id: hal-00884391 \\ https://hal.science/hal-00884391}

Submitted on 1 Jan 1982

HAL is a multi-disciplinary open access archive for the deposit and dissemination of scientific research documents, whether they are published or not. The documents may come from teaching and research institutions in France or abroad, or from public or private research centers.
L'archive ouverte pluridisciplinaire HAL, est destinée au dépôt et à la diffusion de documents scientifiques de niveau recherche, publiés ou non, émanant des établissements d'enseignement et de recherche français ou étrangers, des laboratoires publics ou privés. 


\section{Estimation du coefficient de régression enfant/parent de quelques caractères du pêcher dans le cas de familles issues d'autofécondations}

René MONET \& Yves BASTARD

I.N.R.A., Station d'Arboriculture fruitière, Centre de Recherches de Bordeaux, F 33140 Pont-de-la-Maye

RÉSUMÉ

Pêcher,

Génétique quantitative,

Coefficient de régression,

Autofécondation.
En utilisant les données relevées sur 25 géniteurs et leurs familles issues d'autofécondations, nous avons estimé lc coefficient de régression enfant/parent de quatre caractères agronomiques importants chez le pêcher : date de floraison, date de maturité, poids moyen du fruit, teneur en matière sèche du jus des fruits. Les estimations qui ont été obtenues sont les suivantes :

- Date de floraison : $b_{\mathrm{EP}}=0,90 \pm 0,28$.

- Date de maturité : $b_{\mathrm{EP}}=0,89 \pm 0,10$.

- Poids moyen des fruits : $b_{\mathrm{EP}}=0,60 \pm 0,15$.

- Matière sèche du jus : $b_{\mathrm{EP}}=0,43 \pm 0,33$.

Ces coefficients de régression ont été comparés à ceux obtenus par HANSCHE et al. (1972) sur des familles issues d'hybridations. La comparaison suggère que les interactions de dominance et d'épistasie sont importantes chez le pêcher, bien que cette espèce soit autogame préférenticlle.

\section{SUMMARY}

Peach,

Quantitative genetics, Regression coefficient Self-pollination.

\section{Estimation of offspring-parent regression coefficients of some peach characters in self-pollination families}

From data obtained on 25 progenies from self-pollination of 25 peach varieties, we have estimated the regression coefficient for four characters : date of flowering, date of fruit ripening, average weight of fruits and soluble solids of juice.

The estimates are :

- Date of flowering: $b_{\mathrm{EP}}=0,90 \pm 0,28$.

- Date of maturity: $b_{\mathrm{EP}}=0,89 \pm 0,10$.

- Average weight of fruit : $b_{E P}=0,60 \pm 0,15$.

- Soluble solids of juice : $b_{\mathrm{EP}}=0,43 \pm 0,33$.

These coefficients are compared with those obtained by HANSCHE et al. (1972) for hybrids. The comparison suggests that the interactions of dominance and epistasis are important in peach although this species is mainly autogamous.

\section{INTRODUCTION}

Les études relatives aux caractères quantitatifs sont rares chez le pêcher. Les premiers auteurs ont d'abord traité ces caractères comme s'ils étaient mendéliens simples. Ainsi, CONNORS (1920), parlant du port de l'arbre dans une descendance obtenue par autofécondation de la variété «Elberta », dit ceci : « Cette descendance pourrait donner une disjonction mendélienne 1-2-1 si l'on voulait, mais elle présente actuellement une courbe de Quetelet quand toutes les variations sont enregistrées. »

BLAKE (1933) parle de dominance des caractères « floraison précoce » sur «floraison tardive " et du caractère «petit fruit » sur « gros fruit ».
FRENCH (1951) a été le premier à introduire l'analyse statistique dans l'étude des caractères quantitatifs du pêcher. Il s'intéresse notamment à la date de maturité et l'observe sur les descendances obtenues par autofécondation ou par hybridation de 8 géniteurs. Dans les familles, les individus sont répartis en classes de précocité ; l'auteur calcule ensuite pour chaque famille la valeur moyenne et l'écart-type ; il confronte ces caractéristiques pour les familles issues d'autofécondation et celles issues d'hybridation lorsque les géniteurs sont les mêmes.

Cette étude a été prolongée par BAILEY \& Hough (1959) qui imaginent des gènes de précocité susceptibles de raccourcir la période de maturation d'1, 2 ou 3 semaines. A 
partir de cette hypothèse, ils essaient d'établir le génotype de quelques variétés.

Plus récemment enfin, Hansche et al. (1972) ont estimé l'héritabilité de 10 caractères, à partir d'observations relevées sur un programme d'hybridation faisant intervenir 224 géniteurs et 2300 hybrides. L'héritabilité a été calculée en utilisant la régression enfant/parent moyen.

Nous nous proposons d'estimer ici le coefficient de régression de 4 caractères: date de floraison, date de maturité des fruits, poids moyen des fruits, teneur en matière sèche du jus ; à partir de données relevées sur des familles issues d'autofécondations.

Le coefficient de régression enfant/parent :

$$
b_{E P}=\frac{\operatorname{Cov}_{E P}}{\sigma_{P}^{2}}
$$

est une bonne indication du degré de ressemblance entre parents et enfants; en effet, si la variable aléatoire $E$ mesurée chez les enfants est égale à la variable aléatoire $\mathrm{P}$ mesurée chez les parents, alors :

$$
\operatorname{Cov}_{\mathrm{IPP}}=\sigma_{\mathrm{E}}^{2}=\sigma_{\mathrm{P}}^{2} \Rightarrow \mathrm{b}_{\mathrm{IP}}=1
$$

le coefficient de régression est d'autant plus proche de l'unité que parents et descendants sont semblables pour le caractère étudié.

Dans certaines conditions (population panmictique, formation de couples parentaux par tirage au sort dans cette population), on démontre (FALCONER, 1960) que le coefficient de régression enfant/parent moyen est égal à l'héritabilité du caractère. C'est ainsi que HANSCHE $e t$ al. (1972) ont pu parler d'héritabilité et non de coefficient de régression. Nous ne pouvons assimiler dans notre cas (autofécondations) nos coefficients de régression à l'héritabilité des caractères que nous avons pris en compte. Nous pouvons toutefois comparer légitimement nos coefficients à ceux de ces auteurs, puisque, dans l'un comme dans l'autre cas, ils ont été calculés sur des observations phénotypiques.

Lors de telles estimations, les erreurs interviennent surtout au moment du recueil des données; nous nous efforcerons de montrer quelles sont ces erreurs.

\section{MATÉRIEL ET MÉTHODES}

Dans un but d'analyse des géniteurs, nous avons procédé depuis 1964 à des autofécondations systématiques de variétés de pêcher (MONET, 1977).

Nos familles comprennent de 10 à 280 arbres ; ces différences d'effectifs s'expliquent essentiellement par les difficultés de germination des graines issues de géniteurs à maturité précoce.

Les arbres de ces familles ne sont pas greffés; ils sont plantés à haute densité $(1,50 \mathrm{~m} \times 4 \mathrm{~m})$ et ne subissent qu'une taille d'élagage à partir de la $3^{c}$ année, un éclaircissage sommaire, mais une protection antiparasitaire normalc.

$\mathrm{Au}$ contraire, les géniteurs qui ont donné ces familles sont des variétés greffées, plantées à densité moyenne $(4 \mathrm{~m} \times 4 \mathrm{~m})$; elles sont entretenues en culture intensive : taille annuelle, éclaircissage, traitements réguliers.

Nous avons utilisé les données qui avaient été collectées de 1964 à 1974 sur 25 familles issues d'autofécondations et leurs 25 géniteurs. Ces derniers sont les variétés suivantes : « Babygold 5 », «Babygold 8 », « Babygold 9 », « Carman ", "Cervetto ", "Crimson Gold », « Dixired ", « Fairhaven », « Halehaven », « Henri Moulin », « Loring »,
" Mayflower », « Merrill 49 », « Nectared 6 », " Philp », « Raritan Rose », « Redhaven », « Red June », « Robin »,

« Silver Lode », « Southland », « Springtime », « Suncling », "Suncrest" et «Vivian".

Pour chaque caractère, le coefficient de régression a été estimé par :

$$
b_{\mathrm{EP}}=\frac{\sum(\mathrm{E}-\overline{\mathrm{E}})(\mathrm{P}-\overline{\mathrm{P}})}{\sum(\mathrm{P}-\overline{\mathrm{P}})^{2}}
$$

où

$\mathrm{P}$ valeur du parent,

$\overline{\mathrm{P}}$ moyenne des parents,

E moyenne des valeurs des 10 premiers arbres de chaque famille d'autofécondation,

$\overline{\mathrm{E}}$ moyenne des valeurs $\mathrm{E}$.

La dimension des familles d'autofécondations étant très variable (10 à 280 arbres), nous n'avons retenu dans nos calculs que les valeurs observées sur les 10 premiers arbres de chaque famille. Cela simplifie les calculs et n'entraîne pas de biais dans l'estimation car, à la plantation, la place qu'occupera un arbre est entièrement soumise au hasard.

Les représentations graphiques des droites de régression ont été faites de telle sorte que les axes de coordonnées se coupent à la valeur moyenne du caractère des parents et des enfants.

\section{RÉSULTATS}

\section{A. Coefficient de régression enfant/parent de la date de floraison}

Il est difficile de noter avec précision la date de floraison d'un arbre fruitier car elle est étalée dans le temps. Conventionnellement, on note la date de pleine floraison : c'est le moment où 95 p. 100 environ des fleurs de l'arbre sont ouvertes. Il existe donc une erreur dans la notation de ce caractère qui peut être de 2 ou 3 jours en plus ou en moins de la date réelle.

Il faut signaler aussi que l'observation de la floraison n'a pu être réalisée la même année sur les 25 familles puisque leur mise en place s'est échelonnée sur 10 ans; ceci n'autorise pas à comparer la position relative des familles pour ce caractère. Mais, et ce point est très important, la date de floraison du géniteur a été obtenue la même année où elle a été observée dans la descendance ainsi la valeur du coefficient de régression du caractère reste inaltérée.

La figure 1 illustre la régression enfant/parent pour ce caractère. Sur l'axe horizontal figurent les dates de floraison des parents comptées à partir du $1^{\text {er }}$ mars ; sur l'axe vertical sont représentées les valeurs moyennes de la date de floraison de 10 descendants de chaque famille, comptées elles aussi à partir du $1^{\text {er }}$ mars. Les axes se coupent à la valeur moyenne de tous les parents $(25$ mars) et à la valeur moyenne de toutes les familles d'autofécondations (24 mars). La droite tracée correspond à la régression linéaire enfant/parent ; sa pente est :

$$
\mathrm{b}_{\mathrm{EP}}=0,90 \pm 0,28 \text {. }
$$

C'est un coefficient de régression très élevé. HANSCHE et al. (1972) avaient trouvé pour le même caractère un chiffre beaucoup plus faible $\left(b_{E P}=0,39\right)$. Que faut-il en penser ? Notre coefficient de régression est calculé sur un nombre d'observations moins important que celui de ces auteurs ; il 


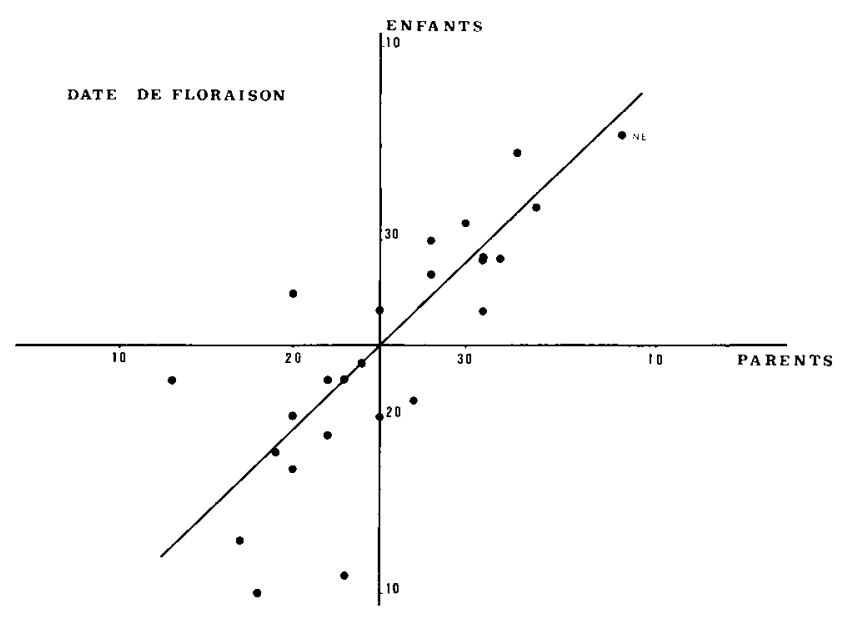

Figure 1

Régression enfant/parent pour la date de floraison; les dates sont comptées à partir du $1^{\text {er }}$ mars. Chaque point a pour abscisse la date de floraison du parent et pour ordonnée la date de floraison moyenne de 10 de ses descendants obtenus par autofécondation.

Offspring-parent regression for flowering date; dates are counted from March Ist. Parent values are shown along the horizontal axis ; mean value for 10 self-pollination offspring along the vertical axis.

a une déviation standard très élevée ; ces faits ne lui sont pas favorables. Cependant, il semble que le chiffre obtenu par HANSCHE et al. soit au contraire sous-estimé puisque celui qu'ils obtiennent pour la date de maturité des fruits $\left(\mathrm{b}_{\mathrm{EP}}=0,84\right)$ est nettement supérieur ; or date de maturité et date de floraison ne sont pas des caractères entièrement indépendants, les ressemblances entre apparentés devraient être peu différentes pour ces 2 caractères.

\section{B. Coefficient de régression enfant/parent de la date de maturité des fruits}

Là encore, il est nécessaire de rappeler la difficulté de noter ce caractère : la maturité d'un arbre est aussi un phénomène échelonné, nous ne notons en fait que le début de maturité lorsque l'on peut trouver sur l'arbre quelques fruits en début de ramollissement. Il est, de plus, probable que le fait que les géniteurs soient des arbres greffés constitue un biais dans notre estimation : le greffage hâte la maturité si le porte-greffe est faible, ou la retarde s'il est vigoureux. Nos géniteurs étant greffés sur le porte-greffe franc de semis «GF 305 », l'effet du porte-greffe est resté vraisemblablement discret.

La figure 2 représente la régression enfant/parent pour ce caractère. Les dates de maturité ont été comptées à partir du $1^{\text {er }}$ juin. La valeur moyenne des dates de maturité des parents et des enfants est le 31 juillet.

La pente de la droite de régression est :

$$
\mathrm{b}_{\mathrm{EP}}=0,89 \pm 0,10 \text {. }
$$

Comme pour la date de floraison, le coefficient de régression est très élevé, mais ici notre résultat est très proche de celui obtenu par HANSCHE et al. $\left(\mathrm{b}_{\mathrm{EP}}=0,84\right)$.

\section{Coefficient de régression enfant/parent du poids moyen des fruits}

Le poids moyen des fruits d'un arbre est largement modifié par les pratiques culturales. Or, nous l'avons déjà

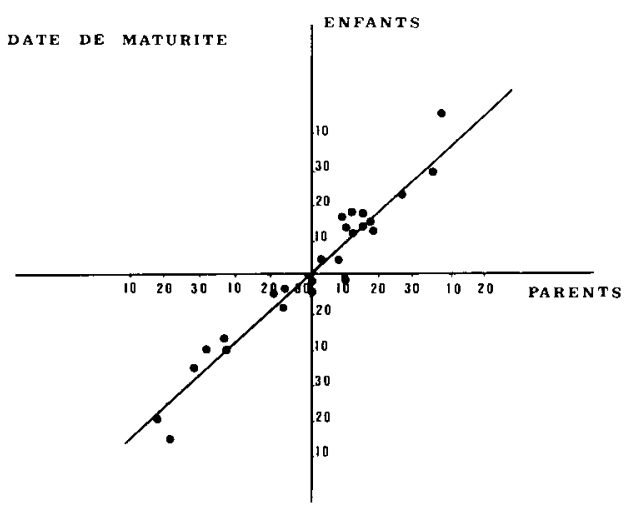

Figure 2

Régression enfant/parent pour la date de maturité du fruit; les dates sont comptées à partir du $1^{\text {er }}$ juin. Chaque point a pour abscisse la date de maturité du parent et pour ordonnée la date de maturité moyenne de 10 de ses descendants obtenus par autofécondation.

Offspring/parent regression for maturity date. Dates are counted from June 1st. Parent values are shown along the horizontal axis; mean value for 10 self-pollination offspring along the vertical axis.

signalé, nos familles d'autofécondations ne bénéficient pas des mêmes traitements que leurs géniteurs; elles sont soumises à des conditions de culture moins favorables : densité élevée, taille sommaire, léger éclaircissage, ce qui fait que le poids des fruits est plus faible qu'il ne serait en verger intensif. Ceci peut accroître les dissemblances entre enfants et parents.

En second lieu, comme il n'est pas possible de peser tous les fruits de chaque arbre pour établir leur poids moyen, on introduit un nouveau biais en ayant recours à l'échantillonnage.

En pratique, le poids moyen des fruits d'un arbre est calculé sur un échantilion de 10 fruits prélevés au hasard dans les différentes zones de la charpente.

La figure 3 représente la régression enfant/parent pour ce caractère. La valeur moyenne de tous les parents est de $154 \mathrm{~g}$, celle des familles d'autofécondation de $119 \mathrm{~g}$.

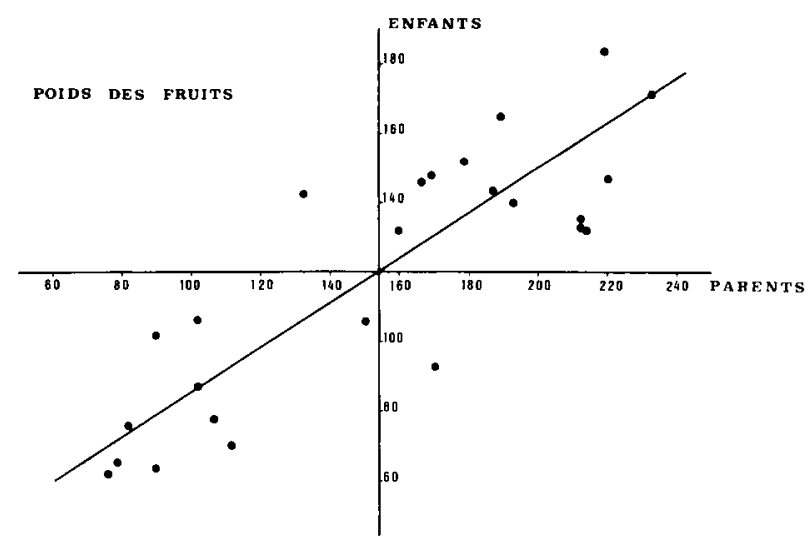

Figure 3

Régression enfant/parent pour le poids moyen du fruit. Les poids moyens sont exprimés en grammes. Chaque point a pour abscisse le poids moyen des fruits du parent et en ordonnée la valeur moyenne du poids des fruits de 10 de ses descendants obtenus par autofécondation.

Offspring/parent regression for mean weight of fruit. Mean weights are expressed in grams. Parent values are shown along the horizontal axis; mean value for 10 self-pollination offspring along the vertical axis. 
La pente de la droite de régression est :

$$
\mathrm{b}_{\mathrm{EP}}=0,60 \pm 0,15 \text {. }
$$

HANSCHE et al. n'avaient pas calculé le coefficient de régression de ce caractère, ils ont estimé celui des 3 dimensions du fruit : longueur du fruit $b_{\mathrm{EP}}=0,31$, distance entre les joues : $b_{E P}=0,26$, distance à la suture : $b_{E P}=0,29$.

Ces coefficients, comparables entre eux, sont plus faibles que celui du poids moyen du fruit que nous avons obtenu.

\section{Coefficient de régression enfant/parent de la teneur en matière sèche du jus des fruits}

La matière sèche soluble contenue dans un jus de pêche est constituée essentiellement par des hydrates de carbone. La teneur en matière sèche d'un jus est donc un caractère intéressant à considérer si l'on veut améliorer la qualité des pêches.

La mesure de ce caractère est facilitée par l'emploi du réfractomètre ; on extrait le jus d'un échantillon de 2 ou 3 fruits par arbre ; quelques gouttes de ce jus sont nécessaires pour connaître l'indice réfractométrique.

Les mêmes réserves qui ont été faites sur la valeur des données utilisées dans le calcul des coefficients de régression du poids moyen des fruits peuvent être rappelées ici : les géniteurs et les descendants n'ont pas été soumis aux mêmes pratiques culturales. La mesure du caractère a été faite sur un échantillon réduit.

Mais, en outre, il faut dire que l'indice réfractométrique se modifiant avec les progrès de la maturation, il eût été nécessaire de faire les mesures sur des échantillons au même état physiologique. Cette condition n'a été qu'imparfaitement réalisée en prélevant le jus de fruits en début de ramollissement.

La figure 4 représente la régression enfant/parent pour ce caractère. La teneur en matière sèche est exprimée en grammes par litre de jus. La valeur moyenne de tous les

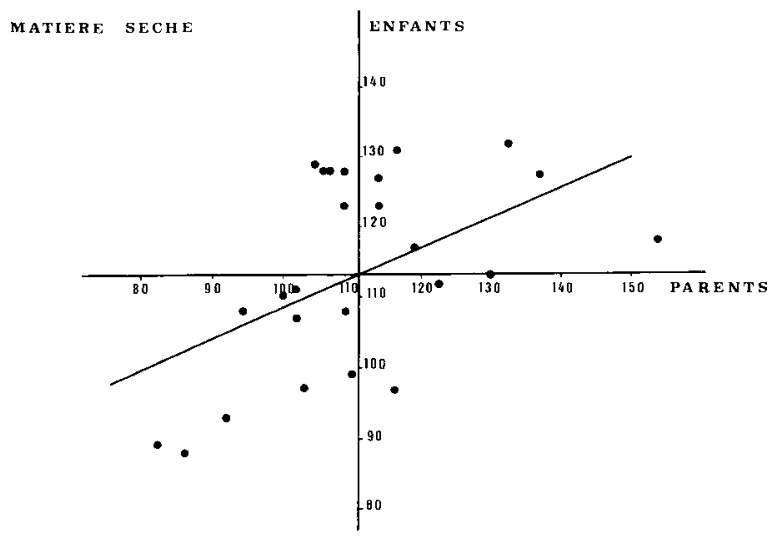

Figure 4

Régression enfant/parent pour la teneur en matière sèche du jus des fruits. La teneur est exprimée en grammes d'équivalents saccharose par litre de jus. Chaque point a pour abscisse la teneur en matière sèche du jus des fruits d'un parent et en ordonnée la valeur moyenne de 10 de ses descendants obtenus par autofécondation.

Offspring-parent regression for soluble solids of fruit juice. The content is expressed in grams of sucrose per liter. Parent values are shown along the horizontal axis and mean value for 10 self-pollination offspring along the vertical axis. parents est de $111 \mathrm{~g} / 1$, celle des enfants est de $113 \mathrm{~g} / \mathrm{l}$. La pente de la régression linéaire est :

$$
\mathrm{b}_{\mathrm{EP}}=0,43 \pm 0,33
$$

Notre coefficient est plus élevé que celui obtenu par HANSCHE et al. $\left(b_{E P}=0,01\right)$; il a aussi une déviation standard très forte.

\section{DISCUSSION ET CONCLUSION}

Le coefficient de régression enfant/parent est une mesure de leur degré de ressemblance ; il permet de prévoir ainsi quel sera l'aspect d'une descendance lorsque l'on utilise tel ou tel géniteur. Nous voyons qu'après un premier cycle d'autofécondations, le degré de ressemblance est très élevé pour la date de floraison et la date de maturité des fruits, il est plus faible pour le poids moyen des fruits et leur teneur en matière sèche.

On peut donc espérer des progrès rapides d'une sélection appliquée à des cycles successifs d'autofécondations, lorsque cette sélection s'exerce sur les deux premiers caractères alors qu'elle sera moins efficace pour les deux derniers.

Ceci nous intéresse plus particulièrement pour le caractère date de floraison puisque nous avons engagé un programme de création de variétés à floraison tardive dans un but d'amélioration de la résistance aux gelées. On peut espérer qu'en autofécondation, la tardiveté se transmettra bien à la descendance et qu'ainsi des progrès rapides seront faits.

En hybridation par contre, si l'on se réfère aux données de HANSCHE et al., nous aurons une moins bonne ressemblance entre parents et enfants pour ce caractère. D'une manière générale d'ailleurs, si l'on compare les coefficients de régression que nous avons obtenus à ceux de ces auteurs, nous constatons que les leurs sont toujours plus faibles. Quelle explication pourrait-on donner à cette observation ? Les coefficients ont été calculés dans chaque cas sur des données phénotypiques qui intègrent des effets génétiques et des effets du milieu.

En supposant que les effets du milieu soient très voisins en France et aux Etats-Unis, ce qui est une hypothèse raisonnable, les différences entre apparentés, plus fortes en hybridation qu'en autofécondation, résultent essentiellement des effets génétiques. L'autofécondation a pour effet de réduire les cas d'interactions géniques (dominance et épistasie); au contraire, l'hybridation les augmente. Par conséquent, les différences des coefficients de régression montrent que, chez le pêcher, il doit exister un effet d'hétérosis important bien que cette espèce soit à autogamie préférentielle.

Toute méthode de sélection qui aura pour but de récupérer cet effet d'hétérosis se justifie donc; notamment en matière de porte-greffes, il faudrait envisager non seulement l'utilisation de lignées de pêchers comme cela se fait actuellement, mais aussi d'hybrides entre certaines de ces lignées pour obtenir une vigueur accrue.

Restent les importantes réserves que nous avons faites concernant la validité de ces résultats, compte tenu des erreurs qui s'accumulent au moment de la collecte des données.

Pour obtenir des résultats plus satisfaisants, il faudrait cultiver les géniteurs et leurs descendants dans un même verger et les soumettre aux mêmes techniques culturales; 
ceci n'est pas aussi facile à obtenir qu'il y paraît puisque les géniteurs étant nécessairement greffés, doivent être préparés 2 ans à l'avance. La mise en place simultanée de tous les géniteurs et de leurs descendants permettrait en outre d'obtenir, pour les caractères fluctuant d'une année à l'autre, une information supplémentaire concernant la position réelle des couples enfant/parent dans la population étudiée, ce qui rendrait la sélection pour un caractère beaucoup plus facile.

Nous n'avons pas, non plus, pour le calcul de la régression, pris en compte l'effet année. Bien que le caractère ait été observé la même année pour chaque couple enfant/parent, les observations des différentes familles se sont échelonnées dans le temps. Cette situation a pour effet d'accroître la valeur du coefficient de régression et explique sans doute en partie la valeur élevée de nos coefficients. Il serait donc nécessaire d'amoindrir l'effet année en utilisant la valeur moyenne du caractère observé plusieurs années sur le parent et les enfants. N'ayant pas d'observations suffisantes, nous n'avons pu effectuer cette correction.

Il faudrait enfin réduire les erreurs dues aux observations et à l'échantillonnage; dans ce domaine les progrès ne peuvent être que modestes.

Reçu le 16 février 1981 Accepté le 25 novembre 1981.

\section{RÉFÉRENCES BIBLIOGRAPHIQUES}

Bailey C. H., Hough L. F., 1959. An hypothesis for the inheritance of season of ripening in progenies from certain early ripening peach varieties and selection. Proc. am. Soc. hortic. Sci., 73, 125-133.

Blake M. A., 1933. The "J. H. Hale" peach as a parent in peach crosses. Proc. am. Soc. hortic. Sci., 29, 131-136.

Connors C. H., 1920. Peach breeding. Summary of results. Proc. am. Soc. hortic. Sci., 17, 108-115.

Falconer D. S., 1960. Introduction to quantitative genetics. The Ronald press company. New York, p. 150-185.
French A. P., 1951. Inheritance of time of ripening and other economic characters. Mass. agric. Exp. Stn, Bull., 462.

Hansche P. E., Hesse C. O., Beres V., 1972. Estimates of genetic and environmental effects on several traits in peach. J. am. Soc. hortic. Sci., 97 (1), 76-79.

Monet R., 1977. Amélioration du pêcher par la voie sexuée ; exposé d'une méthode. Ann. Amélior. Plant., 27 (2), 223-234. 